\title{
Simple models for the distribution of dark matter ${ }^{\star}$
}

\author{
J. An and N. W. Evans
}

Institute of Astronomy, University of Cambridge, Madingley Road, Cambridge CB3 OHA, UK

e-mail: [jin;nwe]@ast.cam.ac.uk

Received 17 June 2005 / Accepted 17 August 2005

\section{ABSTRACT}

We introduce a simple family of models for representing the dark matter in galaxies. The potential and phase space distribution function are all elementary, while the density is cusped. The models are all hypervirial, that is, the virial theorem holds locally, as well as globally. As an application to dark matter studies, we compute some of the properties of $\gamma$-ray sources caused by neutralino self-annihilation in dark matter clumps.

Key words. galaxies: kinematics and dynamics - galaxy: halo - cosmology: dark matter - gamma rays: theory

\section{Introduction}

It is worthwhile to find simple models for the distribution of dark matter in galaxy haloes. This is tantamount to solving the collisionless Boltzmann and Poisson equations for the distribution function $f$, potential $\psi$, and density $\rho$ of the dark matter particles.

Many authors start by assuming a profile for the dark matter density and then solving for the self-consistent potential and distribution function. This has provided some widely-used and notable models for dark matter haloes (e.g., Jaffe 1983; Hernquist 1990). A drawback to this approach is that, even if the density and potential are simple, the distribution function is often unwieldy. For example, the distribution function of the Jaffe (1983) model is a higher transcendental function, while the distribution function of the Hernquist (1990) model is composed of an unwieldy bunch of elementary functions.

It can be advantageous to tackle this problem the other way round, by assuming a simple distribution function (DF) and then solving for the self-consistent potential and the density. Except for Toomre (1982), this reverse approach has not been widely used. Here, we exploit it to build a flexible family of cusped dark matter halo models with an elementary DF and potential (Sects. 2 and 3).

As a simple application, we use our models to study the signal from indirect detection experiments (Sect. 4). In particular, $\gamma$-rays from dark matter annihilation may be identified by forthcoming atmospheric Čerenkov telescopes such as VERITAS ${ }^{1}$

\footnotetext{
* Appendix A is only available in electronic form at http://www. edpsciences.org

1 http://veritas.sao.arizona.edu
}

or by satellite-borne detectors like $G L A S T^{2}$, and so it is useful to have definite predictions from halo models.

\section{A simple distribution function}

\subsection{An ansatz}

Let us assume that the dark halo is spherical, in which case the DF may depend on the binding energy $E$ and the magnitude of the angular momentum $L$. Let us note that the generalized Plummer models, recently studied by Evans \& An (2005) have very simple power-law DFs of the form

$f(E, L) \propto L^{p-2} E^{(3 p+1) / 2}$.

This suggests that it may be worthwhile to look for models with distribution functions given by the sum of such components (cf., Fricke 1952; Toomre 1982);

$f(E, L)=\sum_{i} C_{i} L^{p_{i}-2} E^{\left(3 p_{i}+1\right) / 2}$,

where $C_{i}$ and $p_{i}$ are all constants. Then, the density is of the form

$\rho=\sum_{i} D_{i} r^{p_{i}-2} \psi^{2 p_{i}+1}$

where the constants $D_{i}$ are

$D_{i}=2^{\left(p_{i}+1\right) / 2} \pi^{3 / 2} C_{i} \frac{\Gamma\left(p_{i} / 2\right) \Gamma\left(3 p_{i} / 2+3 / 2\right)}{\Gamma\left(2 p_{i}+2\right)}$.

By integrating the DF over velocity space, it is straightforward to derive the radial and tangential velocity second moments

\footnotetext{
${ }^{2}$ http://www-glast.stanford.edu
} 
$\left\langle v_{r}^{2}\right\rangle$ and $\left\langle v_{\mathrm{T}}^{2}\right\rangle$ respectively. Whereas the anisotropy parameter $\beta=1-\left\langle v_{\mathrm{T}}^{2}\right\rangle /\left(2\left\langle v_{r}^{2}\right\rangle\right)$ is no longer constant in contrast to models of Evans \& An (2005), we still find the remarkably simple relation between the three-dimensional velocity dispersion and the potential;

$\left\langle v_{r}^{2}\right\rangle+\left\langle v_{\mathrm{T}}^{2}\right\rangle=\frac{\psi}{2}$.

In other words, the root mean square velocity is always onehalf of the escape velocity at every spot, or the virial theorem holds locally for any model described by the DFs (2). Evans \& An (2005) coined the term "hypervirial" to describe such stellar dynamical models for which the kinetic energy in each volume element $\left(T=\rho\left\langle v^{2}\right\rangle / 2\right)$ is exactly one-half of the magnitude of the local contribution to the potential energy by the same volume element $(|W|=\rho \psi / 2)$. This idea can be traced back to the classical investigations of Plummer (1911) and Eddington (1916). It has received additional impetus from the recent $N$-body simulations of Sota et al. (2005).

\subsection{Poisson's equation}

While we have established that any spherical system described by a distribution function of the form of Eq. (2) is hypervirial, we still have to find the corresponding density and potential by solving Poisson's equation (here $G=1$ )

$\frac{1}{r^{2}} \frac{\mathrm{d}}{\mathrm{d} r}\left(r^{2} \frac{\mathrm{d} \psi}{\mathrm{d} r}\right)=-4 \pi \sum_{i} D_{i} r^{p_{i}-2} \psi^{2 p_{i}+1}$.

When the sum contains only a single term, this is a particular case of a family of differential equations investigated by Goenner \& Havas (2000), which they called the generalized Lane-Emden equations. In fact, it corresponds to the special case of parameter combinations noted by them (see Eq. (11) of Goenner \& Havas 2000) that permit a rational transformation of variables that leaves the differential equation form-invariant. The one-parameter solution family (Eq. (18) of Goenner \& Havas 2000) is the direct generalization of the Schuster-Emden integral (see e.g. Horedt 1986) and leads to the models of Evans $\&$ An (2005).

Even for the general case when the sum contains more than one terms, the order of Eq. (6) can be reduced in a similar manner (cf., Chandrasekhar 1939). First, let us consider the substitution $\psi=r^{-1 / 2} \varphi=\varphi \exp (-t / 2)$ and $t=\ln r$. Then, the left hand side of Eq. (6) transforms

$$
\frac{1}{r^{2}} \frac{\mathrm{d}}{\mathrm{d} r}\left(r^{2} \frac{\mathrm{d} \psi}{\mathrm{d} r}\right)=r^{-5 / 2}\left(\frac{\mathrm{d}^{2} \varphi}{\mathrm{d} t^{2}}-\frac{\varphi}{4}\right)
$$

while the right hand side can be rewritten using

$$
\sum_{i} D_{i} r^{p_{i}-2} \psi^{2 p_{i}+1}=\sum_{i} D_{i} r^{-5 / 2} \varphi^{2 p_{i}+1}
$$

Hence, Eq. (6) reduces to

$$
\frac{\mathrm{d}^{2} \varphi}{\mathrm{d} t^{2}}=\frac{\varphi}{4}-4 \pi \sum_{i} D_{i} \varphi^{2 p_{i}+1}
$$

which does not involve the independent variable explicitly, and so its order can be reduced by standard techniques (e.g., Ince 1944) to give

$$
\left(\frac{\mathrm{d} \varphi}{\mathrm{d} t}\right)^{2}=A+\frac{\varphi^{2}}{4}\left(1-\sum_{i} 4 B_{i} \varphi^{2 p_{i}}\right),
$$

where $A$ is a constant of integration and

$B_{i}=\frac{4 \pi D_{i}}{p_{i}+1}$

Using the boundary condition at infinity $(\varphi=0$ and $\mathrm{d} \varphi / \mathrm{d} t=0)$, we find that $A=0 .{ }^{3}$ Then, after introducing a further transformation of the variable, $\varphi^{-p}=\vartheta$, equation (10) reduces to

$$
\left(-\frac{2}{p} \frac{\mathrm{d} \vartheta}{\mathrm{d} t}\right)^{2}=\vartheta^{2}-\sum_{i} 4 B_{i} \vartheta^{2\left(1-p_{i} / p\right)}
$$

Here, we note that the differential equation $\left(y^{\prime}\right)^{2}=f(y)$ where $f(y)$ is a polynomial of $y$, the degree of which is at most two, can be solved through elementary functions. However, the right-hand side of Eq. (12) can be a quadratic polynomial of $\vartheta$ if $p=p_{i}$ or $p=2 p_{i}$ for all distinct $p_{i}$ 's. For the simplest case when the sum contains only a single term, it is straightforward to show that either choice of $p$ leads to the solution that reduces to the models of Evans \& An (2005) with the integration constant being the scalelength. The only other possibility is that the sum contains two terms with $p=p_{1}=2 p_{2}$, which is investigated in the following section.

\subsection{Solutions}

For this case, the integration results in

$\vartheta=2 d \cosh \left[\frac{p}{2}\left(t-t_{0}\right)\right]+2 B_{2}=d\left[\left(\frac{r}{r_{0}}\right)^{p / 2}+\left(\frac{r}{r_{0}}\right)^{-p / 2}\right]+2 B_{2}$,

where $t_{0}=\ln r_{0}$ is the integration constant, and $d=\left(B_{1}+B_{2}^{2}\right)^{1 / 2}$. By reinstating $\vartheta=\varphi^{-p}=r^{-p / 2} \psi^{-p}$, we obtain

$$
\frac{1}{\psi^{p}}=d r_{0}^{p / 2}\left[1+2 c\left(\frac{r}{r_{0}}\right)^{p / 2}+\left(\frac{r}{r_{0}}\right)^{p}\right]
$$

where $c=B_{2} / d$. With the normalization $M_{\infty}=1$, we find that $d=r_{0}^{p / 2}$. So, we arrive at a two-parameter $-c$ and $p$ - potential of the form of (incorporating the dimensional constants $G, M$ and $r_{0}$ )

$\psi=\frac{G M}{\left(r_{0}^{p}+2 c r_{0}^{p / 2} r^{p / 2}+r^{p}\right)^{1 / p}}$,

whose distribution function is given by

$f(E, L)=C_{1} L^{p-2} E^{3 p / 2+1 / 2}+C_{2} L^{p / 2-2} E^{3 p / 4+1 / 2}$.

3 The solution with $A \neq 0$ is unphysical because Eq. (10) implies that $(\mathrm{d} \ln \psi / \mathrm{d} \ln r)$ diverges as $r \psi^{2} \rightarrow 0$ unless $A=0$. Nevertheless, we note that it is possible to find explicit solutions with $A \neq 0$ if the sum contains a single term with $p=1 / 2,1$, or 2 . The resulting solutions, expressible in terms of Jacobi or Weierstrass elliptic functions, however, exhibit oscillatory behaviour along the real axis, which we suspect to be a general property of the differential equation. 
Here, the constants $C_{i}$ can be found from Eqs. (4) and (11) with $B_{1}=1-c^{2}, B_{2}=c, p_{1}=p$ and $p_{2}=p / 2$ (henceforth $G=M=r_{0}=1$ ). In order for the distribution function to be non-negative everywhere, we must have $p>0$ and $0 \leq c \leq 1$. In particular, if $c=0$ or $c=1$, the models reduce to those of Evans \& An (2005). We note that the model with $(p, c)=(a, 1)$ is identical to the one with $(p, c)=(a / 2,0)$.

\section{The simple halo models}

Thusfar, we have obtained the gravitational potential (15) corresponding to the simple DF (2). Various limits are already well-known. For example, when $(p, c)=(1,0)$ or $(2,1)$, this is the Hernquist (1990) potential generated by the distribution function first found by Baes \& Dejonghe (2002). When $(p, c)=(2,0)$ or $(4,1)$, this is the isotropic Plummer $(1911)$ model.

The density generated by the potential (15) is

$\rho=\frac{\left(1-c^{2}\right)(p+1) /(4 \pi)}{r^{2-p}\left(1+2 c r^{p / 2}+r^{p}\right)^{2+1 / p}}+\frac{c(p+2) /(8 \pi)}{r^{2-p / 2}\left(1+2 c r^{p / 2}+r^{p}\right)^{1+1 / p}}$,

which can be found from Poisson's equation. Bearing in mind the property (5), we refer to this family as the generalized hypervirial models. Figure 1 shows some typical density profiles. Provided that $c \neq 0$, the second term in Eq. (17) is dominant when $r \rightarrow 0\left[\rho \sim r^{-(2-p / 2)}\right]$ and $r \rightarrow \infty\left[\rho \sim r^{-(3+p / 2)}\right]$. If $p \leq 2$, the density is monotonically decreasing outwards-radially regardless of the value of $c$. If $2<p<4$, there may be a region of increasing density depending on the value of $c$, although the model still exhibits a cuspy centre. The $p=4$ model is cored whereas there is a hole at the centre if $p>4$.

Notice that the density profile - although composed of entirely elementary functions - is a bit more complicated than either the potential or the DF. We argue that this is the right way round as most applications will use the potential (for example, for integrating the orbits in numerical simulations) or the DF (for example, for calculating the flux of dark matter particles on a detector). It is much more useful to have models with simple potentials and DFs than those with simple density profile.

Evidence from $N$-body simulations suggests that the density profile of the dark halo follows a simple functional form. One of the most commonly cited examples is that of Navarro et al. (1995, 1996, henceforce NFW), which is basically a double power-law characterized by $r^{-1}$ cusp at the centre and $r^{-3}$ fall-off at large radii. Since every member of the generalized hypervirial models has a finite mass, none of them can reproduce the $r^{-3}$ density fall-off - which implies an infinite mass - in the outer region. Regarding the behavior in the inner region, however, many members of the family indeed exhibit a $r^{-1}$-like cusp, including the well-known example of the Hernquist (1990) model. In fact, the additional freedom afforded by the parameter $c$ admits more flexibility in the behavior around the scale length. For example, we find that the model with $(p, c)=(2,3 / 4)$ provides a better fit to the NFW profile witin a scalelength than the Hernquist (1990) model. Actually, if we allow a slight deviation of the cusp slop, there exists a

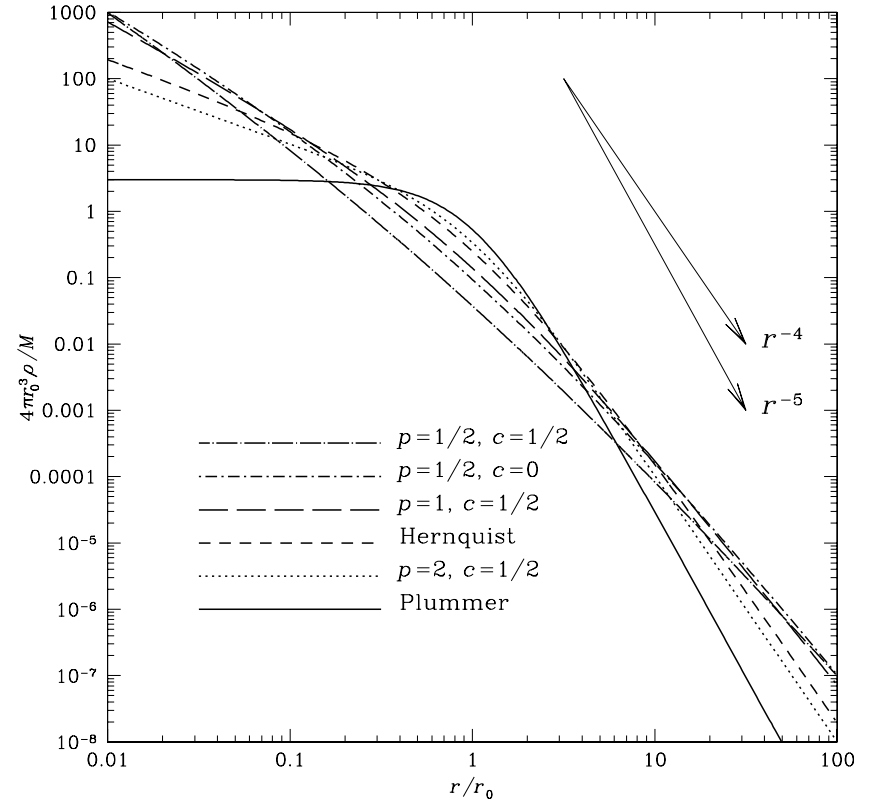

Fig. 1. The density profile of the generalized hypervirial models. The Plummer and Hernquist models are included in the generalized hypervirial family as the cases $(p, c)=(2,0)$ [or $(4,1)]$ and $(p, c)=(1,0)$ [or $(2,1)]$ respectively. Note that the model with $(p, c)=(1 / 2,0)$ is the same as $(p, c)=(1,1)$.

trade-off between varying $p$ and $c$ which produces very similar behaviour of the density profiles in the inner parts.

The circular speed and the cumulative mass can be found as

$$
\begin{aligned}
& v_{\mathrm{c}}^{2}=-r \frac{\mathrm{d} \psi}{\mathrm{d} r}=\frac{c r^{p / 2}+r^{p}}{\left(1+2 c r^{p / 2}+r^{p}\right)^{1 / p+1}}, \\
& M_{r}=r v_{\mathrm{c}}^{2}=\frac{1+c r^{-p / 2}}{\left(1+2 c r^{-p / 2}+r^{-p}\right)^{1 / p+1}} .
\end{aligned}
$$

Here, the total mass is finite and therefore the circular speed falls off as Keplerian at large radii $\left(v_{\mathrm{c}} \sim r^{-1 / 2}\right)$. Figure 2 shows plots of the circular velocity as a function of $r / r_{1 / 2}$, where $r_{1 / 2}$ is the half-mass radius, (i.e., $M_{r_{1 / 2}}=1 / 2$ ). The models with inner density slopes $\rho \propto r^{-1}$ or $\rho \propto r^{-3 / 2}$ have rotation curves similar to those of the Navarro et al. $(1995,1996)$ and Moore et al. (1998) models and so are flattish over a wide range of radii.

The velocity dispersions are

$$
\begin{aligned}
& \left\langle v_{r}^{2}\right\rangle=\frac{1+c^{2}+c g}{2\left(1+c^{2}+c g\right)+p(2+c g)} \psi, \\
& \left\langle v_{\mathrm{T}}^{2}\right\rangle=\frac{p}{2} \frac{2+c g}{2\left(1+c^{2}+c g\right)+p(2+c g)} \psi,
\end{aligned}
$$

where $g=r^{p / 2}+r^{-p / 2}$ and therefore the anisotropy parameter varies according to

$$
\beta=1-\frac{p}{4}\left(\frac{2+c g}{1+c^{2}+c g}\right)
$$

From its construction, the hypervirial relation is automatically satisfied. Because the potential is everywhere finite, 


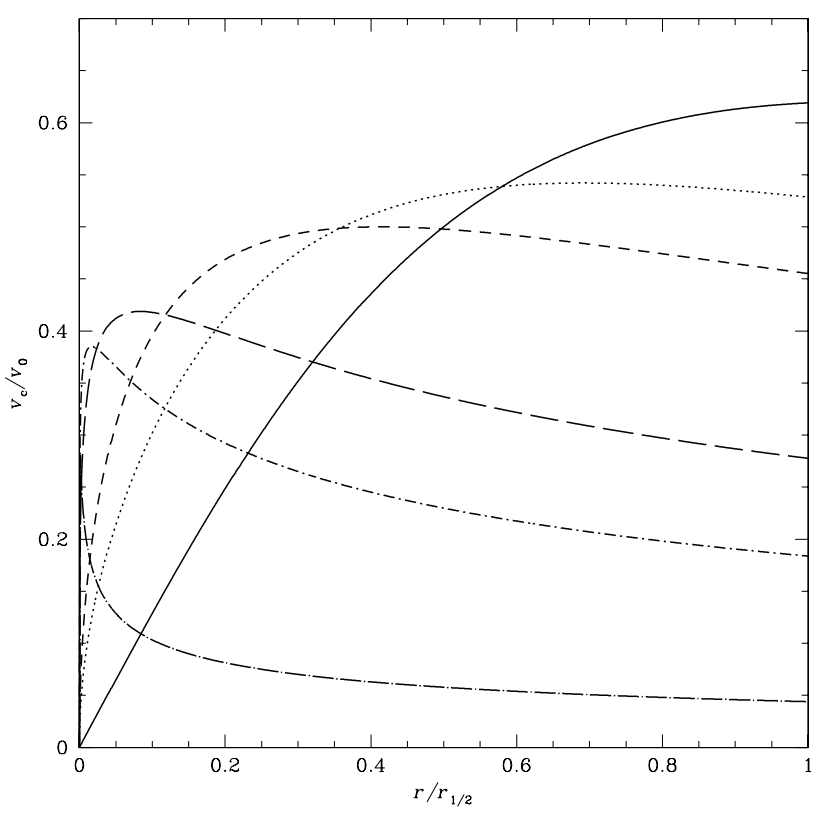

Fig. 2. The circular velocity curves of the generalized hypervirial models as a function of $r / r_{1 / 2}$, where $r_{1 / 2}$ is the half-mass radius. Here, $v_{0}^{2}=G M / r_{0}$. The line types are as in Fig. 1 .

the hypervirial relation also implies that the velocity dispersions are everywhere finite. In particular, assuming $c \neq 0$, the central velocity dispersions are $\left\langle v_{r}^{2}\right\rangle=1 /(p+2)$ and $\left\langle v_{\mathrm{T}}^{2}\right\rangle=$ $p /[2(p+2)]$. In addition,

$\frac{\mathrm{d} \beta}{\mathrm{d} r}=\frac{p^{2} c\left(1-c^{2}\right) r^{p / 2-1}\left(r^{p}-1\right)}{8\left(c+r^{p / 2}\right)^{2}\left(1+c r^{p / 2}\right)^{2}}$,

and therefore, provided that $0<c<1$, the anisotropy parameter $\beta$ decreases from $\beta=1-p / 4$ at $r=0$ to $\beta=1-p /[2(1+c)]$ at $r=1$ and then increases back to $\beta \rightarrow 1-p / 4$ as $r \rightarrow \infty$. In other words, in this model, the velocity dispersions are more radially anisotropic near the centre and the outskirts whereas they are relatively less radially anisotropic around the region of the radial scalelength. However, we also note that the model as a whole always possesses a more radially biased velocity dispersion than the isotropic model unless $p>2(1+c)$.

It has been suggested that dark matter haloes achieve an almost isotropic state near the centre and become more and more radially anisotropic in the outer parts, at least according to cosmological $N$-body simulations (Hansen \& Moore 2005). Our DFs are radially anisotropic and therefore better suited to modelling the outer parts and envelopes of dark haloes. They are unsuitable for the class of problems in which central anisotropy plays a critical role.

\section{An application: the distribution of $\gamma$-ray sources}

Diemand et al. (2005) have presented evidence from numerical simulations that dark matter may be clumped into minihaloes of Earth mass and larger. They estimate that $\sim 50 \%$ of the total mass of the dark matter halo is bound to dark matter substructures. These objects may be detectable by virtue of the $\gamma$-rays from neutralino annihilation in the very centres of the clumps. Diemand et al. (2005) also point out that the nearest mini-haloes will be amongst the very brightest sources from neutralino annihilation and may be found either with the forthcoming GLAST satellite or next-generation atmospheric Čerenkov telescopes as high proper motion, discrete $\gamma$-ray sources.

If this idea is correct, then there are some immediate consequences. First, because of the offset of the Sun's location from the centre of the dark halo, the distribution of such $\gamma$-ray sources is anisotropic and the magnitude of the anisotropy is an indicator of the cusp slope at the Galactic Centre. This effect is already well-known in studies of halo origin of the ultrahigh energy cosmic rays (e.g., Evans et al. 2000). Let us use Galactic coordinates $(s, \ell, b)$, where $s=|s|$ is heliocentric distance, and $(\ell, b)$ are Galactic longitude and latitude. Then, the relative number density of $\gamma$-ray sources is

$F(\ell, b) \propto \int s^{2} \mathrm{~d} s w(s) \rho\left[\boldsymbol{R}_{\odot}+\boldsymbol{s}_{\ell, b}\right]$

where $\boldsymbol{R}_{\odot}$ is the Galactocentric solar position and $w(s)$ is the selection function. For bright sources, the selection function is proportional to the relative luminosity and so $w(s) \propto s^{-2}$. The density profile is truncated at a radius at which the dark matter annihilation rate matches the collapse timescale of the cusp. With this assumption, a tiny constant density core is created (e.g., Tyler 2002) and convergence of the integral (24) is guaranteed. The overall normalisation of the integral depends on the fraction of dark matter bound in mini-haloes, as opposed to smoothly distributed dark matter. The anisotropy effect is clearly illustrated in Fig. 3, which shows Hamer-Aitoff projections in equatorial coordinates for two of the halo models. The first is a model with $\rho \propto r^{-1}$ at the centre $(p, c)=(2,1 / 2)$, and second a model with $\rho \propto r^{-7 / 4}$ at the centre $(p, c)=(1 / 2,1 / 2)$. The more highly cusped the model, the greater the anisotropy. If there are enough detections, the magnitude of the anisotropy can be quantified by harmonic analysis (see e.g., Evans et al. 2000).

Second, if the high proper motion sources can be identified, this will provide the first direct evidence on the velocity distribution of the dark matter. It is therefore useful to have predictions of the velocity distributions. The proper motion and radial velocity distribution of dark matter clumps can be calculated easily for our models, because the DFs are simple. The proper motion and radial velocity distributions in direction $\mathrm{d}^{2} \Omega=\cos b \mathrm{~d} \ell \mathrm{d} b$ is

$\frac{\mathrm{d}^{2} N_{\text {obs }}}{\mathrm{d} v_{\ell} \mathrm{d} v_{b}}\left(v_{\ell}, v_{b}, \ell, b\right)=\mathrm{d}^{2} \Omega \iint \mathrm{d} v_{s} \mathrm{~d} s f(E, L) s^{2} w(s)$,

$\frac{\mathrm{d} N_{\text {obs }}}{\mathrm{d} v_{s}}\left(v_{s}, \ell, b\right)=\mathrm{d}^{2} \Omega \iiint \mathrm{d} v_{\ell} \mathrm{d} v_{b} \mathrm{~d} s f(E, L) s^{2} w(s)$.

Here, the velocity of the dark matter particles has been resolved into components $\left(v_{s}, v_{\ell}, v_{b}\right)$ based on Galactic coordinates while $f(E, L)$ is the distribution function of the dark matter. For models with DFs (2), the above triple integrals are easily computed via Gaussian quadrature. In fact, the second moments of these distributions are entirely analytic, as sketched out in Appendix A. Figure 4 shows the proper motion and line of sight velocity distributions for the dark matter clumps in three directions. When looking towards the Galactic Centre or anti-Centre 

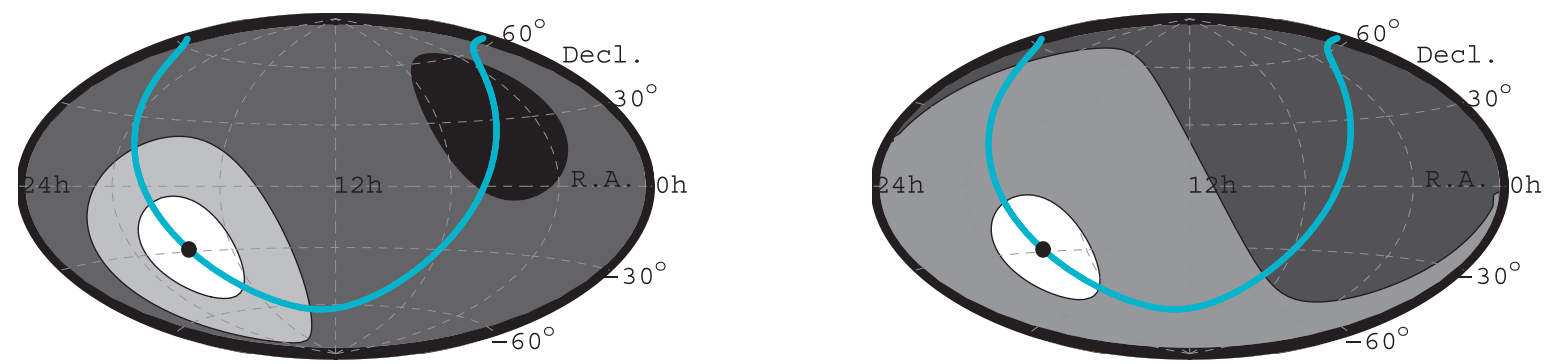

Fig. 3. Contour plots of the (projected) number density of $\gamma$-ray sources in equatorial coordinates. The left hand panel refers to a model with $\rho \propto r^{-1}$ at the centre $(p, c)=(2,1 / 2)$, while the right-hand panel to a model with $\rho \propto r^{-7 / 4}$ at the centre $(p, c)=(1 / 2,1 / 2)$. The grey-scale gives the relative number density, with white representing the highest values and black the lowest. The number density changes by a factor of 10 on moving from one contour to the next. The Galactic centre and the Galactic plane are marked.
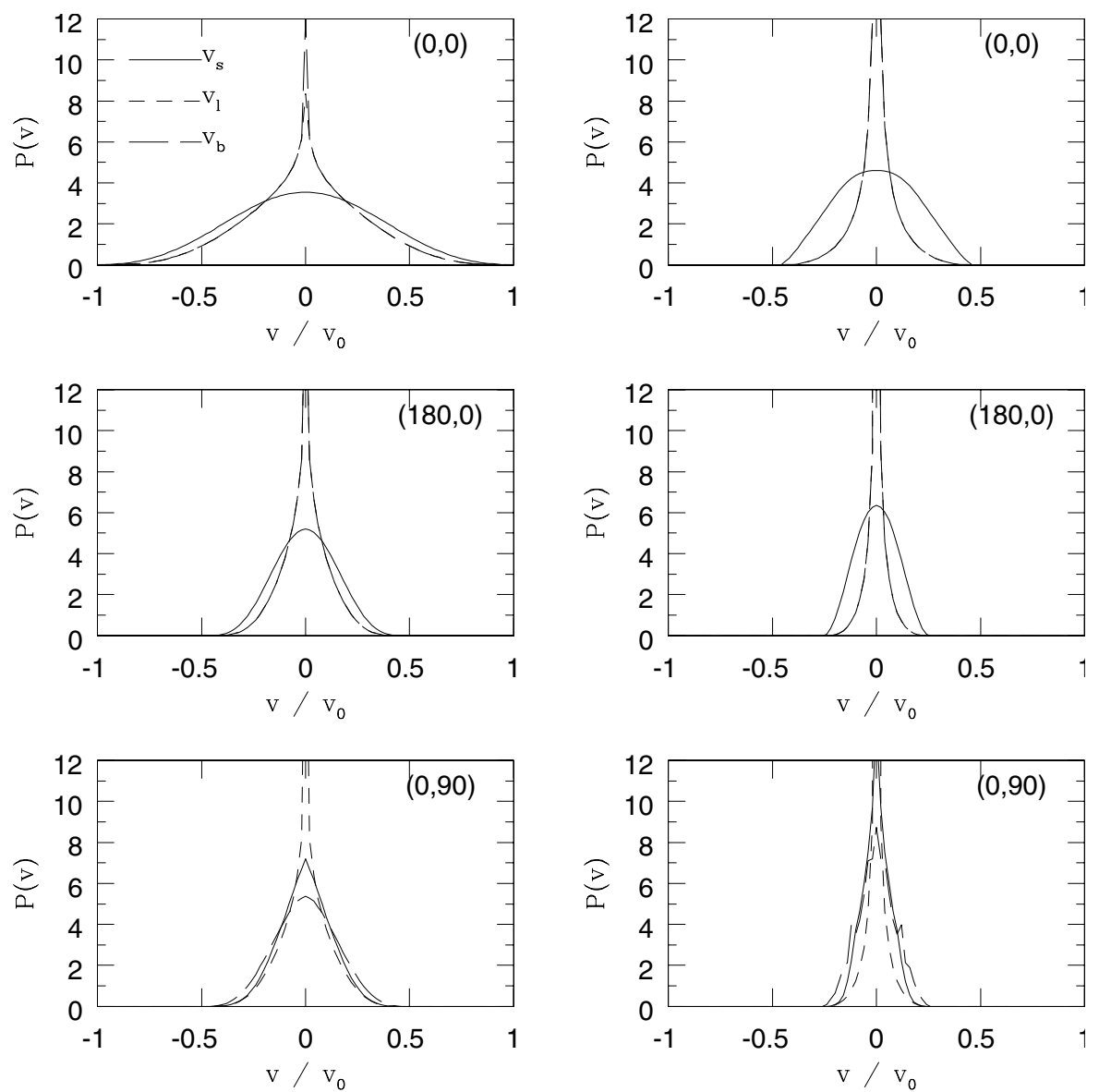

Fig. 4. The distribution of line of sight velocities and proper motions of dark matter particles towards the Galactic Centre (upper panels), the anti-Centre (middle panels) and the Galactic North Pole (lower panels). The left-hand panels refer to the model with $(p, c)=(2,1 / 2)$, while the right-hand panels refer to the model with $(p, c)=(1 / 2,1 / 2)$. The velocity is given in units of $v_{0}=\left(G M / r_{0}\right)^{1 / 2}$.

in spherical models, then the distributions of the two tangential velocity components $v_{\ell}$ and $v_{b}$ are the same, though this is not necessarily the case in other directions. All three velocity distributions show very significant deviations from Gaussianity. The sources with highest tangential velocity occur towards the the Galactic Centre direction and it is therefore in this direction that GLAST or VERITAS may find it easiest to detect them unambiguously.

Although these facts have been established in the context of our simple family of models, we argue that they are likely to be generic. The anisotropy effect in source positions is a consequence of the Sun's offset from the center. The fastest moving sources are likely to be found in the direction in which the gravitational potential well is deepest.

\section{Conclusions}

We have presented a simple family of halo models useful for the study of dark matter haloes. They have a simple potential and distribution function, being composed of two terms of the form terms like $L^{p-2} E^{(3 p+1) / 2}$ - where $L$ is the total 
angular momentum, $E$ the binding energy and $p$ is some constant. As an application, we have presented the properties of discrete $\gamma$-ray sources arising from neutralino annihilation in dark matter mini-haloes. This suggestion for the composition of the dark matter is derived the numerical simulations of Diemand et al. (2005). If true, then nearby, high proper motion discrete $\gamma$-rays sources may be detectable by forthcoming missions such as GLAST. We have shown that there is an anisotropy in the positions of such $\gamma$-ray sources because of the offset of the Sun from the Galactic Centre. We have also provided distributions of proper motions and line of sight velocities of such sources. The best direction in which to look is towards the Galactic Centre $(\ell, b)=(0,0)$. There are more sources in this direction and they have the highest proper motion.

This paper also continues the study of hyperviriality begun by Eddington (1916) and further developed by Evans \& An (2005). In spherical symmetry, any model which has a distribution function that is a sum of terms like $L^{p-2} E^{(3 p+1) / 2}$ is hypervirial. This leads to the derivation of a general differential equation that any spherical hypervirial system must satisfy. We have solved it to find the most general models, for which the potential can be written down in terms of elementary functions.

We briefly note that, in the case of axisymmetry, any model which has a distribution function that is a sum of terms like $L_{z}^{p-2} E^{(3 p+1) / 2}-$ where $L_{z}$ is the angular momentum component about the axis of symmetry - is also hypervirial. It is possible to derive the equation for the corresponding potential and demonstrate that at least two analytical solutions exist, corresponding to the flattened Plummer model studied by Lynden-Bell (1962) and a particular case among the prolate galaxy models of Lake (1981). In fact, only the Plummer model - the sole isotropic, hypervirial model - can be generalized to give an axisymmetric hypervirial system. It is an open question whether any further hypervirial generalizations of the Plummer model can be found.

\section{References}

Baes, M., Dejonghe, H. 2002, A\&A, 393, 485

Chandrasekhar, S. 1939, An Introduction to the Study of Stellar Structure. (Chicago IL: The University of Chicago Press)

Diemand, J., Moore B., Stadel, J. 2005, Nature, 433, 389

Eddington, A. S. 1916, MNRAS, 76, 572

Evans, N. W., An, J. 2005, MNRAS, 360, 492

Evans, N. W., Ferrer, F., Sarkar, S. 2000, Astropart. Phys., 17, 319

Fricke, W. 1952, Astron. Nachr., 280, 193

Goenner, H., Havas, P. 2000, J. Math. Phys., 41, 7029

Hansen, S. H., \& Moore, B. 2005, New Astron., in press [arXiv:astro-ph/0411473]

Hernquist, L. 1990, ApJ, 356, 359

Horedt, G. P. 1986, A\&A, 160, 148

Ince, E. L. 1944, Ordinary Differential Equations (New York NY: Dover)

Jaffe, W. 1983, MNRAS, 202, 995

Lake, G. 1981, ApJ, 243, 111

Lynden-Bell, D. 1962, MNRAS, 123, 447

Moore, B., Governato, F., Quinn, T., Stadel, J., Lake, G., 1998, ApJ, 499, L5

Navarro, J. F., Frenk, C. S., \& White, S. D. M. 1995, MNRAS, 275, 720

Navarro, J., Frenk, C. S., White, S. D. M. 1996, ApJ, 462, 563

Plummer, H. C. 1911, MNRAS, 71, 460

Sota, Y., Iguchi, O., Morikawa, M., Nakamichi, A. 2005, Proc. of the $\mathrm{CN}$-Kyoto International Workshop on Complexity [arXiv: astro-ph/0508308]

Toomre, A. 1982, ApJ, 259, 535

Tyler, C. 2002, Phys. Rev. D, 66, 023509 


\section{Online Material}




\section{Appendix A: Second moments of the velocity distributions}

It is straightforward to compute the six independent components of the velocity dispersion tensor in a Galactic coordinate system. These formulae do not appear to have been given before, and so we quote them here:

$$
\begin{aligned}
\left\langle v_{s}^{2}\right\rangle= & \left\langle v_{x}^{2}\right\rangle \cos ^{2} b \cos ^{2} \ell+\left\langle v_{y}^{2}\right\rangle \cos ^{2} b \sin ^{2} \ell+\left\langle v_{z}^{2}\right\rangle \sin ^{2} b \\
& +\left\langle v_{x} v_{y}\right\rangle \cos ^{2} b \sin 2 \ell-\left\langle v_{y} v_{z}\right\rangle \sin 2 b \sin \ell \\
& -\left\langle v_{x} v_{z}\right\rangle \sin 2 b \cos \ell, \\
\left\langle v_{b}^{2}\right\rangle= & \left\langle v_{x}^{2}\right\rangle \sin ^{2} b \cos ^{2} \ell+\left\langle v_{y}^{2}\right\rangle \sin ^{2} b \sin ^{2} \ell+\left\langle v_{z}^{2}\right\rangle \cos ^{2} b \\
& +\left\langle v_{x} v_{y}\right\rangle \sin ^{2} b \sin 2 \ell+\left\langle v_{y} v_{z}\right\rangle \sin 2 b \cos \ell \\
& +\left\langle v_{x} v_{z}\right\rangle \sin 2 b \sin \ell, \\
\left\langle v_{\ell}^{2}\right\rangle= & \left\langle v_{x}^{2}\right\rangle \sin ^{2} \ell+\left\langle v_{y}^{2}\right\rangle \cos ^{2} \ell-\left\langle v_{x} v_{y}\right\rangle \sin 2 \ell, \\
\left\langle v_{s} v_{b}\right\rangle= & -\left\langle v_{x}^{2}\right\rangle \cos b \sin b \cos { }^{2} \ell-\left\langle v_{y}^{2}\right\rangle \cos b \sin b \sin ^{2} \ell \\
& +\left\langle v_{z}^{2}\right\rangle \cos b \sin b-\left\langle v_{x} v_{y}\right\rangle \sin 2 b \cos \ell \sin \ell \\
& -\left\langle v_{x} v_{z}\right\rangle \cos 2 b \cos \ell-\left\langle v_{y} v_{z}\right\rangle \cos 2 b \sin \ell,
\end{aligned}
$$$$
\left\langle v_{\ell} v_{s}\right\rangle=\left\langle v_{y}^{2}-v_{x}^{2}\right\rangle \cos b \sin \ell \cos \ell+\left\langle v_{x} v_{y}\right\rangle \cos b \cos 2 \ell
$$$$
\text { (A.1) }\left\langle v_{z}^{2}\right\rangle=\frac{z^{2}}{r^{2}}\left\langle v_{r}^{2}\right\rangle+\frac{x^{2}+y^{2}}{r^{2}}\left\langle v_{\theta}^{2}\right\rangle
$$
(A.4) obtain the second moments of the observable distributions (the$$
\left\langle v_{x} v_{y}\right\rangle=\frac{x y}{r^{2}}\left(\left\langle v_{r}^{2}\right\rangle-\left\langle v_{\theta}^{2}\right\rangle\right),
$$

$$
\left\langle v_{y} v_{z}\right\rangle=\frac{y z}{r^{2}}\left(\left\langle v_{r}^{2}\right\rangle-\left\langle v_{\theta}^{2}\right\rangle\right)
$$

Here, $\left\langle v_{\theta}^{2}\right\rangle=\left\langle v_{\phi}^{2}\right\rangle=\left\langle v_{\mathrm{T}}^{2}\right\rangle / 2$. Hence, by substituting in the known velocity dispersions in spherical polars (Eqs. (20) and (21)), we line of sight velocities and the proper motions).

)

(5)$$
+\left\langle v_{x} v_{z}\right\rangle \sin b \sin \ell-\left\langle v_{y} v_{z}\right\rangle \sin b \cos \ell,
$$

$$
\begin{aligned}
\left\langle v_{\ell} v_{b}\right\rangle= & \left\langle v_{x}^{2}-v_{y}^{2}\right\rangle \sin b \sin \ell \cos \ell-\left\langle v_{x} v_{y}\right\rangle \cos b \cos 2 \ell \\
& +\left\langle v_{x} v_{z}\right\rangle \cos b \sin \ell-\left\langle v_{y} v_{z}\right\rangle \cos b \cos \ell
\end{aligned}
$$

